\title{
CFR: A Finite Volume Approach for Computing Incompressible Viscous Flow
}

\author{
A.B. Harichandan ${ }^{1 \dagger}$ and A. Roy ${ }^{2}$ \\ ${ }^{1}$ Research Scholar, Department of Aerospace Engineering, IIT Kharagpur, Kharagpur-721302, India \\ ${ }^{2}$ Assistant Professor, Department of Aerospace Engineering, IIT Kharagpur, Kharagpur-721302, India \\ $\dagger$ Corresponding Author Email: harichandan.iitkgp@gmail.com
}

(Received June 9, 2010; accepted July 21, 2010)

\begin{abstract}
An incompressible unsteady viscous two-dimensional Navier-Stokes solver is developed by using "Consistent Flux Reconstruction" method. In this solver, the full Navier-Stokes equations have been solved numerically using a collocated finite volume scheme. In the present investigation, numerical simulations have been carried out for unconfined flow past a single circular cylinder with both structured and unstructured grids. In structured grid, quadrilateral cells are used whereas triangular elements are used in unstructured grid. Simulations are performed at Reynolds number $(R e)=100$ and 200. Flow simulation over a NACA0002 airfoil at $R e=1000$ using unstructured grid based solver is also reported. The vortex shedding phenomena is mainly investigated in the flow. It is observed that the nature of flow depends strongly on the value of the Reynolds number. The present results are found to be in satisfactory agreement with several numerical results and a few experimental results available from literature.
\end{abstract}

Keywords: Finite volume Navier Stokes solver, Incompressible flow, Circular cylinder, Vortex shedding, Structured grid, Unstructured grid, Strouhal number, Airfoil

\section{INTRODUCTION}

Finite-volume methods ensure conservativeness of mass and momentum equations and are more accurate than finite-difference methods in which coordinate transformation of the governing equations from the physical domain to the computational domain is required. In finite-volume methods, no coordinate transformation is necessary. Hence most of the fluid problems involving complex boundaries are solved using finite volume discretization schemes. In incompressible flow calculations satisfaction of the mass and momentum conservation laws is important in obtaining the velocity and pressure fields. Pressure field required for momentum equation is calculated such that velocities calculated from momentum equations should satisfy the continuity equation. This step is called 'Flux Reconstruction step' and it should have at least second order accuracy while avoiding spurious pressure modes. As there is no equation readily available for the calculation of pressure, it must be derived from the continuity equation by using the divergence-free constraint. For this, 'pressure-Poisson' equation obtained by combining the discretized momentum equations with the discretized continuity equation is solved either by 'pressure correction method' or 'pressure-Poisson method'.

Over the decades, many researchers have developed different schemes for computing incompressible viscous flows. Rhie and Chow (1983) brought forth the pioneering work in the context of using staggered grid by retaining the velocity components and solving the problems in the physical plane. In this approach, designated as 'Physical Interpolation Approach' (PIA), the cell face velocities are obtained by solving the momentum equations at each of the cell faces. This ensures the pressure-velocity coupling between the adjoining cells and prevents the odd-even pressure oscillations. However, this way of calculating the cell face velocities needs matrix inversion because of implicit scheme and hence requires long computing time. Choi et al. (1993) refined the momentum interpolation scheme of Rhie and Chow (1983) to employ curvilinear co-variant velocity components as cell face velocities. Relative performance of the two schemes was examined through applications to several test problems and it was observed that the solver with co-variant velocity components provides good convergence and stable behavior even on strongly non orthogonal grids. Choi et al. (1993) compared two finite volume calculation methods for incompressible flows on non orthogonal grids with different grid arrangements, one with conventional staggered arrangement and another with non staggered arrangement of Rhie \& Chow (1983). Around the same time, Deng et al. (1994) developed an explicit scheme called 'Consistent Physical Interpolation' (CPI) for the calculation of cell face velocities and used these values with an implicit scheme for the solution of momentum equations at the cell centers. This method does not 
require any matrix inversion for calculating the cell face velocities and is therefore relatively less expensive than Rhie and Chow (1983) method. Later Roy and Bandyopadhyay (2006) developed a technique which uses explicit calculation for both cell face centre flux reconstruction and cell centre momentum equation updating, which has eliminated the tedious matrix inversion procedures. Chakrabarty (1990) developed a vertex-based finite-volume solution of the twodimensional Navier-Stokes equations. The method was applied to solve flow past NACA 0012 airfoil and the obtained results have been compared with the existing numerical and experimental results. In vertex-based solution, the nodal point discretization, where the flow quantities are ascribed to the corners of the cell, gave better accuracy for the highly stretched and skewed grids. Unlike cell-centered schemes, the surface boundary conditions are satisfied exactly at the vertices along the body surface and the pressure on the wall are computed directly by this scheme. McBride et al. (2007) developed a finite volume method for the solution of flow on distorted meshes. The method investigates a range of cell-centered, vertex based and hybrid approaches to finite volume discretization of the Navier-Stokes equations. Recently, Ding et al. (2007) applied 'mesh-free least square-based finite difference (MLSFD) method' to numerically simulate the flow field around two circular cylinders arranged in side-byside and tandem configurations. For each configuration, with various geometrical arrangements, simulations have been performed in the low Reynolds number range, at $R e=100$ and $R e=200$. Nishikawa (2007) developed a multigrid algorithm for the third-order accurate solution of Cauchy-Riemann equations discretized in the cell-vertex finite-volume fashion. The solution values are stored at vertices and the residuals defined on triangular elements. The resulting method gave sufficiently accurate solutions on relatively coarse grids. Combined with a multigrid technique, the method gave rise to a highly accurate and efficient solver. The method demonstrates its accuracy and efficiency on both structured and unstructured triangular grids. Sang and $\mathrm{Li}$ (2007) constructed a multi-layer hybrid grid method to simulate complex flow field around two dimensional (2-D) and three dimensional (3-D) configurations. The method combines Cartesian grids with structured grids and triangular meshes. A cellcentered finite volume flow solver was developed for computing both inviscid and viscous flows by solving Euler and Navier-Stokes equations.

Recently, there has been significant progress in the area of incompressible flow calculations with unstructured finite volume method. Mathur and Murthy (1997) presented a finite volume pressure-based method on unstructured mesh for multidimensional incompressible flows. A multigrid scheme was used to solve the discretised equations. The scheme was shown to perform satisfactorily with a variety of quadrilateral/hexahedral, triangular/tetrahedral, and hybrid meshes. Mathur and Murthy (1997) also presented a methodology for incorporating pressure boundary conditions in the context of a collocated numerical scheme for incompressible flows. Raithby (1999) described the use of finite volume method on unstructured meshes which can be used for heat transfer applications in addition to fluid flow solution. Kang and Kim (2002) developed a pressure-based, unstructured, finite-volume method to resolve complex reacting flows accurately. Woodfield and Suzuki (2003) presented a vertex-centered, three-dimensional, unstructured finite volume method on Cartesian coordinates and applied to number of test cases involving incompressible flow at low Reynolds numbers. Perron et al. (2004) proposed a new method to solve the Navier-Stokes equations for incompressible viscous flow using a finite volume method on unstructured meshes. The governing equations were discretized using a collocated, cellcentered arrangement of velocity and pressure with the solution variables being stored at the cell circumcentres. Dalal et al. (2008) proposed a new cell-centered finite volume method for unsteady solutions on complex geometries. Both two-dimensional and threedimensional Navier-Stokes equations were solved for incompressible laminar flow on unstructured meshes. The above discussed calculation techniques are extremely useful in solving incompressible flow past various complex geometries.

Several researchers have conducted extensive experiments on flow past bluff body geometries. One of the important experimental works was performed by Perry et al. (1982). They studied the flow using variety of flow visualization techniques. A sequence of instantaneous stream line patterns have been obtained using photography of the motion of aluminum particles suspended in the flow. Nakamura et al. (1996) made experimental measurements and numerical simulations on elongated rectangular cylinder at $R e=200-10^{3}$. It was observed that there exist two types of vortex shedding processes: one is the Von-Karman alternate shedding and the other is the impinging shear layer instability type where one separated shear layer can be unstable in the presence of sharp trailing edges at high Reynolds number. Cheng and Liu et al. (2000) made experimental studies on square cylinder at Reynolds numbers ranging from 2000 to 21000 at varying incidences. Measurement of vortex shedding frequencies and surface pressures has been reported. It was observed that there exists rapid increase in Strouhal number at an incidence of $13^{\circ}$ for Reynolds number greater than 5300 . Reason for that is described as the strong pressure recovery on lower side face of the cylinder which is associated with flow reattachment.

In the present scheme, the authors have adopted the numerical procedure proposed by Roy and Bandyopadhyay (2006) for solving flow past two dimensional arbitrary body geometries. In this scheme, the cell face center velocities are reconstructed explicitly by solving the momentum equations on flux reconstruction control volumes defined judiciously around the respective cell face centers. This is followed by solution of the cell centre pressure field using a discrete Poisson equation developed from the reconstructed velocities and updating the cell centre velocities by using an explicit scheme. In the present paper, an incompressible 2-D finite volume laminar Navier-Stokes solver based on structured (O-grid) and unstructured (triangular elements) grid is reported. The solver is based on body-fitted curvilinear collocated grid. It has been applied to solve unconfined flow past a 
circular cylinder. The simulations are carried out at $R e$ $=100$ and 200. The grid independence of the results is studied in detail for structured grid. Also, numerical simulation of flow past a NACA0002 airfoil at $R e=$ 1000 using unstructured grid based solver is performed. The numerical results have been validated with results available from literature. The comparison is satisfactory.

\section{GOVERNING EQUATIONS}

The equations governing incompressible viscous fluid flow in two-dimensions are the continuity equation and the two components of momentum equation. In absence of body forces and heat transfer, these equations can be expressed in the conservative non-dimensional primitive variable form as follows:

Continuity equation:

$$
\frac{\partial \mathrm{u}}{\partial \mathrm{x}}+\frac{\partial \mathrm{v}}{\partial \mathrm{y}}=0
$$

Momentum equations:

x-momentum:

$$
\begin{aligned}
& \frac{\partial u}{\partial t}+\frac{\partial\left(u^{2}\right)}{\partial x}+\frac{\partial(u v)}{\partial y} \\
& =-\frac{\partial p}{\partial x}+\frac{1}{\operatorname{Re}} \times\left(\frac{\partial^{2} u}{\partial x^{2}}+\frac{\partial^{2} u}{\partial y^{2}}\right)
\end{aligned}
$$

y-momentum:

$$
\begin{aligned}
& \frac{\partial \mathrm{v}}{\partial \mathrm{t}}+\frac{\partial(\mathrm{uv})}{\partial \mathrm{x}}+\frac{\partial\left(\mathrm{v}^{2}\right)}{\partial \mathrm{y}} \\
& =-\frac{\partial \mathrm{p}}{\partial \mathrm{y}}+\frac{1}{\operatorname{Re}} \times\left(\frac{\partial^{2} \mathrm{v}}{\partial \mathrm{x}^{2}}+\frac{\partial^{2} \mathrm{v}}{\partial^{2}}\right)
\end{aligned}
$$

where the velocity components $\mathrm{u}$ and $\mathrm{v}$ are in the $\mathrm{x}$ and $\mathrm{y}$-directions respectively, $\mathrm{p}$ is ratio of pressure and density, $R e$ is the Reynolds number and $t$ is the non dimensional time.

\section{Finite Volume Discretization of GOVERNING EQUATIONS}

To obtain a numerical solution, the governing flow equations are discretized by a finite volume technique based on the integral form of the equations to be solved. The discretization of the governing equations for structured grid is done in the same manner as was proposed by Roy and Bandyopadhyay (2006). Therefore, the discritization procedure for unstructured grid is described here. The physical region, in which the equations are solved, is divided into elementary triangular cells within which the integration is performed. Only the coordinates of the corners of the cells are necessary. In the present investigation unstructured triangular grid has been generated using GAMBIT $6.2 .1^{\circledR}$ software and used as input to the solver. For any arbitrary triangular cell as shown in Fig. 1, Eqs. (1), (2a) and (2b) can be integrated over the control volume and hence discretized. The explicit forward Euler method has been used for the discretizing the time derivative which is first order accurate in time.

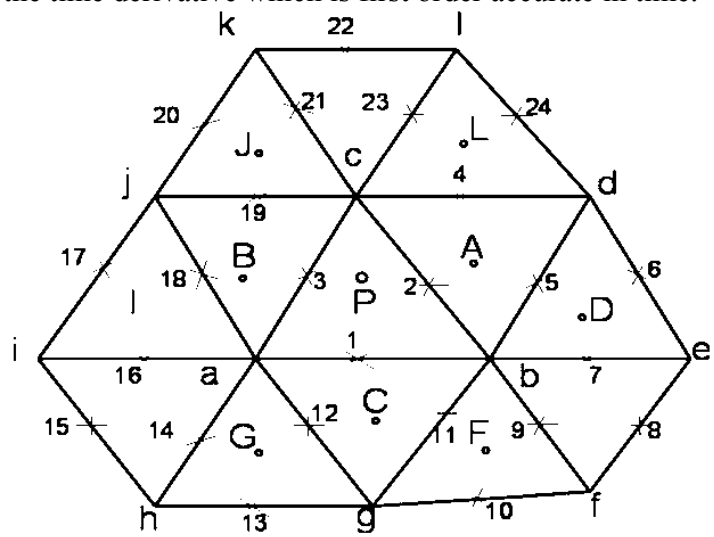

Fig. 1. Grid arrangement showing the collocated triangular main control volume.

Since very small time steps have been used for all calculations, this does not compromise the time accuracy to a significant extent.

\section{APPLICATION OF CFR SCHEME ON UNSTRUCTURED GRID}

Based on the above finite volume discretization of the governing equations, an explicit two-dimensional solver has been developed. The solver makes use of collocated grid arrangement, where the flow variables $\mathrm{u}, \mathrm{v}$ and $\mathrm{p}$ share the same location at the centre of the cells. For the calculation of the convective and pressure fluxes through the cell faces, the unknown values (i.e. $u_{1}, u_{2}$, $\mathrm{u}_{3}, \mathrm{v}_{1}, \mathrm{v}_{2}, \mathrm{v}_{3}, \mathrm{p}_{1}, \mathrm{p}_{2}$ and $\mathrm{p}_{3}$ ) at the centre of the cell faces need to be evaluated.

The cell face-centre velocities are obtained by using a CFR scheme based on triangular cells. The present approach involves the solution of the $\mathrm{x}$ and $\mathrm{y}$ components of momentum equations at the centre of the faces of the each cell. This provides the solution for the required cell face-centre velocities $\mathrm{u}_{1}, \mathrm{v}_{1}, \mathrm{u}_{2}, \mathrm{v}_{2}, \mathrm{u}_{3}$ and $v_{3}$ for flux calculation. These values are then substituted into the discrete continuity equation to obtain the discrete Poisson equation for pressure. In order to maintain the accuracy of the finite volume discretization, the cell face velocities are approximated by a second-order accurate closure method. The cell face-centre pressures are obtained by linearly interpolating the cell centre pressure values calculated by solving the pressure-Poisson equation.

When the cell face velocities are obtained by linear interpolation, the cell face velocity e.g. $\mathrm{u}_{1}$, on the face ' 1 ', comes as a function of the cell centre values of the u-velocity component of the concerned cell and its neighbours, but is independent of the corresponding vvelocity component and pressure. Although upwind interpolation schemes can be used to circumvent the numerical instability problems, spurious pressure modes exist when such linear interpolation formulae are implemented on collocated grids. One of the most effective means to overcome this difficulty is to use a physically consistent flux reconstruction approach by which the cell face velocities are expressed not only in 


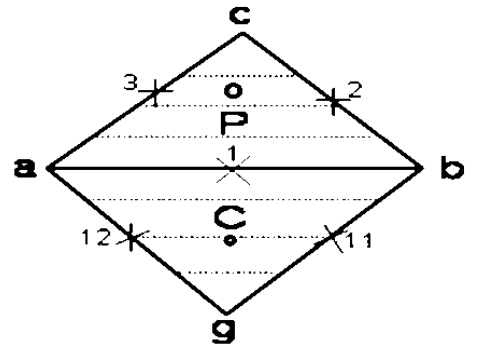

Fig. 2. Grid arrangement showing the control volumes for calculation of cell face velocities at 1, 2 and 3 faces for triangular grid arrangement.

terms of the dependent variable $u$, in this case, but also other physical quantities $\mathrm{v}$ and $\mathrm{p}$. In the present solver, a fully explicit scheme is adopted for the reconstruction of cell face velocities as well as for updating the flow variables at the cell centers. A 10-point stencil is used for both pressure and velocity calculations. For calculating the viscous fluxes on the face ' 1 ' (flux reconstruction cell centered about ' 1 ', comprising of cells $\mathrm{P}$ and $\mathrm{C}$ Fig. 2), the values of velocities at points ' $a$ ', ' $g$ ', ' $b$ ' and 'c' are necessary. For updating the flow variables at the cell centers, the momentum equations are solved in an explicit manner. The layout of the flux reconstruction cells used in the present solver is different from that of Roy and Bandyopadhyay (2006). The present flux reconstruction cells are chosen in such a manner that the integration points for the main control volume and the reconstruction control volume coincide; therefore it reduces the computational effort.

The closures of the cell face velocities $u_{1}, v_{1}, u_{2}, v_{2}, u_{3}$ and $v_{3}$ and are obtained from the discretized $u$ and $v-$ components of the momentum equations at the points ' 1 ', ' 2 ' and ' 3 ' respectively (Fig. 2). The finite volume schemes used at these points are similar to that used at point ' $\mathrm{P}$ '. The discretizations of the various terms in the $\mathrm{x}$-component of momentum equation are given as follows:

Unsteady term:

$\iint_{\Omega_{1}}\left(\frac{\partial \mathrm{u}}{\partial \mathrm{t}}\right)_{1} \mathrm{~d} \Omega_{1}=\left(\frac{\partial \mathrm{u}}{\partial \mathrm{t}}\right)_{1} \times \mathrm{a}_{1}$

$=\left(\frac{\mathrm{u}_{1}^{\mathrm{n}+1}-\mathrm{u}_{1}^{\mathrm{n}}}{\Delta \mathrm{t}}\right) \times\left(\mathrm{a}_{\mathrm{P}}+\mathrm{a}_{\mathrm{C}}\right)$

Convective term:

$$
\begin{aligned}
& \iint_{\Omega_{1}}\left(\frac{\partial(\mathrm{uu})}{\partial \mathrm{x}}+\frac{\partial(\mathrm{vu})}{\partial \mathrm{y}}\right)_{1} \mathrm{~d} \Omega_{1} \\
& =\oint_{\mathrm{C}_{1}}((\mathrm{uu}) \times \mathrm{dy}-(\mathrm{vu}) \times \mathrm{dx}) \\
& =\mathrm{u}_{11}^{2} \times \Delta \mathrm{y}_{\mathrm{gb}}-\mathrm{u}_{11} \times \mathrm{v}_{11} \times \Delta \mathrm{x}_{\mathrm{gb}} \\
& +\mathrm{u}_{2}^{2} \times \Delta \mathrm{y}_{\mathrm{bc}}-\mathrm{u}_{2} \times \mathrm{v}_{2} \times \Delta \mathrm{x}_{\mathrm{bc}} \\
& +\mathrm{u}_{3}^{2} \times \Delta \mathrm{y}_{\mathrm{ca}}-\mathrm{u}_{3} \times \mathrm{v}_{3} \times \Delta \mathrm{x}_{\mathrm{ca}} \\
& +\mathrm{u}_{12}^{2} \times \Delta \mathrm{y}_{\mathrm{ag}}-\mathrm{u}_{12} \times \mathrm{v}_{12} \times \Delta \mathrm{x}_{\mathrm{ag}} \\
& +\mathrm{O}\left(\left|\mathrm{r}_{12}\right|^{3}, \ldots \ldots . .\right)=\mathrm{UCFLUX} \mathrm{X}_{1}
\end{aligned}
$$

Pressure term:

$$
\begin{aligned}
& \iint_{\Omega_{1}}\left(\frac{\partial \mathrm{p}}{\partial \mathrm{x}}\right)_{1} \mathrm{~d} \Omega_{1}=\oint_{\mathrm{C}_{1}} \mathrm{pdy} \\
& =\mathrm{p}_{11} \times \Delta \mathrm{y}_{\mathrm{gb}}+\mathrm{p}_{2} \times \Delta \mathrm{y}_{\mathrm{bc}} \\
& +\mathrm{p}_{3} \times \Delta \mathrm{y}_{\mathrm{ca}}+\mathrm{p}_{12} \times \Delta \mathrm{y}_{\mathrm{ag}} \\
& =\mathrm{UPFLUX}_{1}
\end{aligned}
$$

Diffusive term:

$$
\begin{aligned}
& \iint_{\Omega_{1}}\left[\frac{\partial}{\partial \mathrm{x}}\left(\frac{\partial \mathrm{u}}{\partial \mathrm{x}}\right)+\frac{\partial}{\partial \mathrm{y}}\left(\frac{\partial \mathrm{u}}{\partial \mathrm{y}}\right)\right]_{1} \mathrm{~d} \Omega_{1} \\
& =\oint_{\mathrm{C}_{1}}\left[\left(\frac{\partial \mathrm{u}}{\partial \mathrm{x}}\right) \times \mathrm{dy}-\left(\frac{\partial \mathrm{u}}{\partial \mathrm{y}}\right) \times \mathrm{dx}\right] \\
& =\left(\frac{\partial \mathrm{u}}{\partial \mathrm{x}}\right)_{11} \times \Delta \mathrm{y}_{\mathrm{gb}}+\left(\frac{\partial \mathrm{u}}{\partial \mathrm{x}}\right)_{2} \times \Delta \mathrm{y}_{\mathrm{bc}} \\
& +\left(\frac{\partial \mathrm{u}}{\partial \mathrm{x}}\right)_{3} \times \Delta \mathrm{y}_{\mathrm{ca}}+\left(\frac{\partial \mathrm{u}}{\partial \mathrm{x}}\right)_{12} \times \Delta \mathrm{y}_{\mathrm{ag}} \\
& -\left(\frac{\partial \mathrm{u}}{\partial \mathrm{y}}\right)_{11} \times \Delta \mathrm{x}_{\mathrm{gb}}-\left(\frac{\partial \mathrm{u}}{\partial \mathrm{y}}\right)_{2} \times \Delta \mathrm{x}_{\mathrm{bc}} \\
& -\left(\frac{\partial \mathrm{u}}{\partial \mathrm{y}}\right)_{3} \times \Delta \mathrm{x}_{\mathrm{ca}}-\left(\frac{\partial \mathrm{u}}{\partial \mathrm{y}}\right)_{12} \times \Delta \mathrm{x}_{\mathrm{ag}} \\
& +\mathrm{O}\left(\left|\mathrm{r}_{12}\right|^{3}, \ldots \ldots . .\right)=\mathrm{UDFLUX}{ }_{1}
\end{aligned}
$$

In Eq. (3a), the 'lumped mass' approach has been applied to the flux reconstruction cell on the face ' 1 ' of the cell ' $\mathrm{P}$ '. The area of the reconstruction cell is the sum of the areas of cells ' $\mathrm{P}$ ' and ' $\mathrm{C}$ '. The value of the variable at the reconstruction cell centre, namely $\mathrm{u}_{1}$, is used as the representative value for the entire reconstruction cell for evaluating the transient term. $\Omega_{1}$ is the domain of the ' 1 ' cell (with ' 1 ' as centre and a-g$\mathrm{b}-\mathrm{c}$ as the four nodes of the reconstruction cell as shown in Fig. 2) and $C_{1}$ is the contour enclosing it. The values of the properties at various nodal points like $\mathrm{a}, \mathrm{b}$, c, etc. are obtained by linear interpolation of neighbouring cell centre property values. In Eqs. (3b), (3c) and (3d); the surface integral of the terms has been changed to line integral by using Green's theorem which is calculated over the reconstructed cell with centre as ' 1 ' and a-g-b$\mathrm{c}$ as the four nodes of the contour $\mathrm{C}_{1}$ enclosing it.

The first order velocity derivatives $(\partial \phi / \partial x)$ and $(\partial \phi / \partial y)$ at points $2,3,11$ and 12 are obtained using Taylor series expansion. The velocity derivatives at the other integration points and on other faces (2 and 3) are calculated using similar formulae. The resulting expression for $\mathrm{u}_{1}$ at the $(\mathrm{n}+1)^{\text {th }}$ time level is: 
$\mathrm{u}_{1}^{\mathrm{n}+1}=\mathrm{u}_{1}^{\mathrm{n}}+\frac{\Delta \mathrm{t}}{\left(\mathrm{a}_{\mathrm{P}}+\mathrm{a}_{\mathrm{C}}\right)}$

$\times\left(-\right.$ UCFLUX $_{1}-$ UPFLUX $_{1}+$ UDFLUX $\left._{1}\right)$

The cell face velocity at $n^{\text {th }}$ time level i.e. $u_{1}^{n}$ is taken as the linear interpolation of the adjoining nodal values at that time level. In a similar manner the flux closure relationships for $\mathrm{v}_{1}, \mathrm{u}_{2}, \mathrm{v}_{2}, \mathrm{u}_{3}$ and $\mathrm{v}_{3}$ respectively are also obtained at the $(n+1)$ th time level.

\section{The Pressure poisson Equation}

The equation for pressure is obtained by substituting the expressions for $\mathrm{u}_{1}^{\mathrm{n}+1}, \mathrm{v}_{1}^{\mathrm{n}+1}, \mathrm{u}_{2}^{\mathrm{n}+1}, \mathrm{v}_{2}^{\mathrm{n}+1}, \mathrm{u}_{3}^{\mathrm{n}+1}$ and $\mathrm{v}_{3}^{\mathrm{n}+1}$ into the discrete continuity equation. The following pressure-Poisson equation is obtained with pressure as unknown:

$p_{P}=\frac{1}{C_{P}} \times\left[\begin{array}{l}\text { SOURCE }-C_{A} \times p_{A} \\ -C_{B} \times p_{B}-C_{C} \times p_{C} \\ -C_{D} \times p_{D}-C_{F} \times p_{F} \\ -C_{G} \times p_{G}-C_{I} \times p_{I} \\ -C_{J} \times p_{J}-C_{L} \times p_{L}\end{array}\right]$

where the coefficients $\mathrm{C}_{\mathrm{P}}, \mathrm{C}_{\mathrm{A}}, \mathrm{C}_{\mathrm{B}}, \mathrm{C}_{\mathrm{C}}, \mathrm{C}_{\mathrm{D}}, \mathrm{C}_{\mathrm{F}}, \mathrm{C}_{\mathrm{G}}$ $, \mathrm{C}_{\mathrm{I}}, \mathrm{C}_{\mathrm{J}}$ and $\mathrm{C}_{\mathrm{L}}$ are the geometrical parameters of the cell and SOURCE is the total source term comprising of the cell divergence at the $n^{\text {th }}$ time level, $D_{i}^{n}$, and the convective and diffusive fluxes at the cell faces. The term SOURCE is an explicit function of the nodal variables $\mathrm{u}_{\mathrm{i}}$ and $\mathrm{v}_{\mathrm{i}}$ which include the concerned cell and its nine neighbours as follows:

Equation (5) is used directly as the pressure equation to determine the pressure field. For the cells near the body boundary, the expression for the pressure equation is obtained by applying the zero velocity boundary condition in the discretized continuity equation. Zero normal pressure gradient across the body boundary is applied in Eq. (5). Pressure on the body boundary is obtained by using the Neumann boundary condition and special care is taken to satisfy the compatibility condition (Abdallah 1987) in the discretized pressurePoisson equation for such cells. Satisfaction of the compatibility condition ensures that there is zero net source term when the discretized equations over the entire computational domain are considered. Dirichlet boundary condition of free-stream values has been applied in the outer boundary of the flow domain. Gauss-Siedel method has been used for the iterative solution of the pressure-Poisson equation. Once the pressure-Poisson equation is solved, the cell centre pressure values are available. The cell face-centre pressures are obtained by linear interpolation of adjacent cell centre values. The reconstructed cell centre momentum equations are obtained by substituting the values of velocity derivatives at cell face-centers, interpolated cell centre pressures, and the values of the cell face-centre velocities obtained by the CFR approach as formulated in the present investigation into the discretized momentum equations. From these equations, the velocities at the cell centers are calculated and updated in time explicitly.

\section{INITIAL CONDITION AND BOUNDARY CONDITION}

The governing equations for the viscous incompressible flow are mixed parabolic-elliptic in nature. The equations are parabolic with respect to time and elliptic with respect to space. This means that the solution marches forward in time due to the parabolic behaviour and disturbances may travel along any direction, upstream or downstream due to the elliptic bahaviour. Therefore initial conditions need to be set at the beginning of the solution and boundary conditions surrounding the domain should be specified.

In the beginning of the solution process, uniform freestream velocity and pressure field are prescribed in each cell of the flow domain as given below:

$\left.\begin{array}{l}\mathrm{u}[\mathrm{i}]=\mathrm{u}_{\infty} \\ \mathrm{v}[\mathrm{i}]=\mathrm{v}_{\infty} \\ \mathrm{p}[\mathrm{i}]=\mathrm{p}_{\infty}\end{array}\right\}$ for all triangular cells

This physically means that the body is suddenly introduced into a uniform free-stream flow. Free-stream parameters are indicated by suffix $\infty$. In the present calculations unconfined flow past a single circular cylinder has been considered. For the rectangular domain, the inlet, outlet, top boundary and bottom boundary are kept far away from the body surface. At all the outer boundaries, Dirichlet boundary condition is applied with respect to the free-stream values. On the body surface, no-slip condition is used.

\section{Results AND Discussion}

Flow past an isolated circular cylinder has the attractive features like vortex shedding behind the cylinder and the periodic variation of the flow field at moderate Reynolds number. These vortices get convected and diffused into the cylinder wake forming the well known Karman vortex street. As the geometry of the flow as well as the initial and boundary conditions are symmetric, the Navier-Stokes equations should produce symmetric solution even at moderate Reynolds numbers $(R e>40)$. However, the truncation and round-off errors as well as errors produced due to the numerical approximation scheme act as perturbing factors and eventually trigger the vortex shedding phenomenon.

In this study, the CFR method is applied to simulate flows around a single circular cylinder within the low Reynolds number range, i.e. $R \mathrm{e}=100$ and 200 . In this numerical simulation, streamlines and vorticity contours are plotted as the flow visualization aids. Some flow parameters characterizing the flow aspects such as lift and drag coefficients and Strouhal number are also computed and quantitatively compared with the results of other researchers.

The present results are compared with some of the numerical and experimental results available from literature for validating the CFR scheme. Figure 3 shows the close-up view of the structured O-grid and unstructured triangular grid generated around a circular cylinder. 


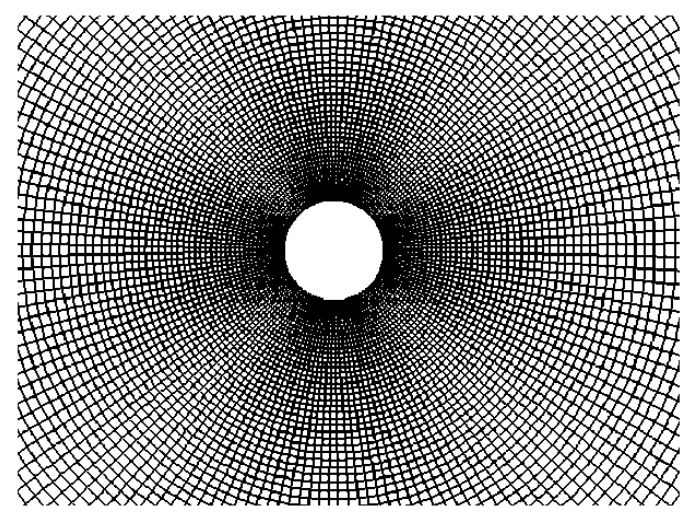

Structured O-grid

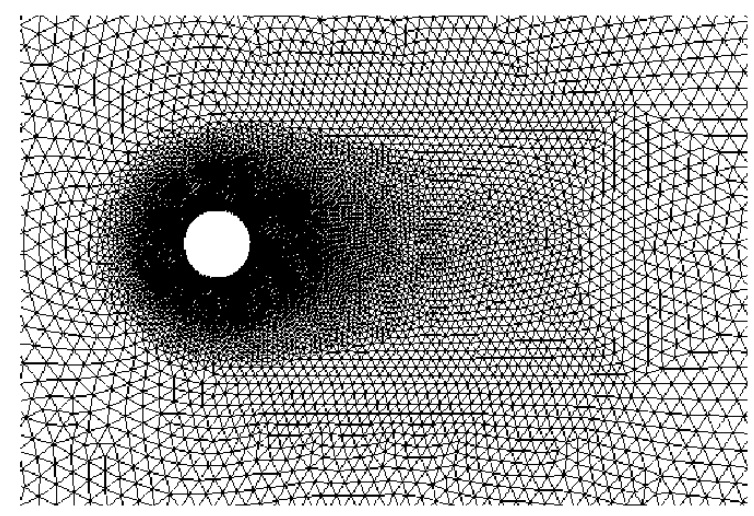

Unstructured triangular grid

Fig. 3. Close-up view of the grid generated around a circular cylinder.
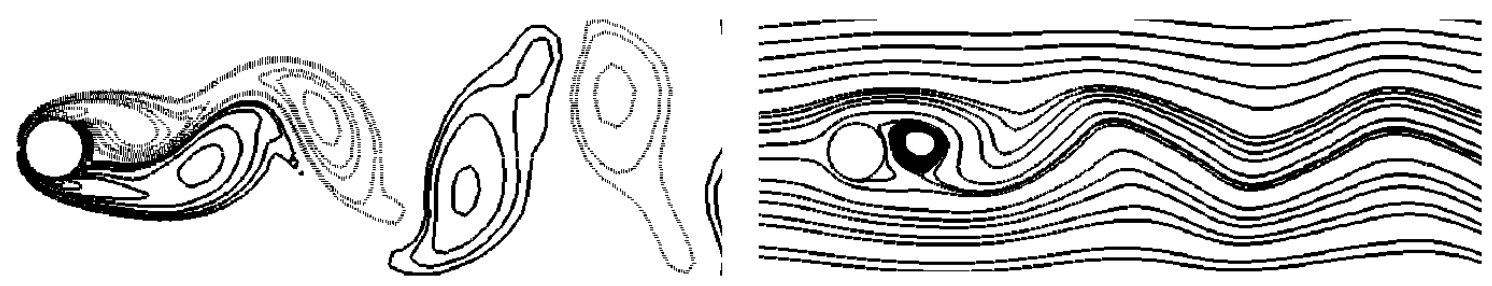

$$
\operatorname{Re}=100
$$

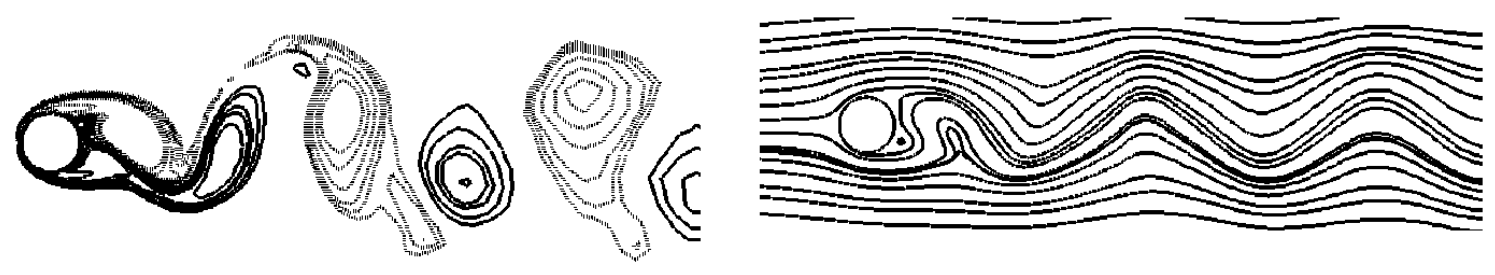

$\operatorname{Re}=200$

Fig. 4. Vorticity contours and streamlines of flow past a circular cylinder.

\subsection{Numerical Simulation of Flow Past A Circular Cylinder Using Structured Grid Based Solver}

In the present investigation, flow past a circular cylinder has been computed at $R e=100$ and 200. The overall characteristics like the lift and drag coefficients and Strouhal number are used for the validation of the scheme. The overall lift and drag coefficients are obtained from the contributions of body surface pressure and shear stress. The present computation has been carried out using a $160 \times 120$ O-grid generated around a circular cylinder using Laplace equation. The outer boundary is kept at 30D (D is the diameter of the cylinder) from the centre of the cylinder. This grid is used as input to the structured grid based Navier-Stokes solver which uses the body-fitted curvilinear grid. The size of the grid was decided based on a grid independence study carried out on four different grids at $R e=200$. Table 1 gives the details of the grid independence test.Based on the variation of the values of lift coefficients, drag coefficients and Strouhal number, Grid 3 has been chosen for the flow calculations.

Table 1 Grid independence test carried out at $R e=200$.

\begin{tabular}{|c|c|c|c|}
\hline Grid size & $\mathrm{CL}$ & $\mathrm{CD}$ & $\begin{array}{c}\text { Strouhal } \\
\text { number }\end{array}$ \\
\hline $60 \times 40($ Grid 1$)$ & \pm 0.21 & $0.80 \pm 0.048$ & 0.190 \\
\hline $100 \times 80($ Grid 2$)$ & \pm 0.35 & $0.845 \pm 0.05$ & 0.192 \\
\hline $160 \times 120($ Grid 3$)$ & \pm 0.487 & $1.484 \pm 0.05$ & 0.198 \\
\hline $200 \times 160($ Grid 4$)$ & \pm 0.51 & $1.508 \pm 0.05$ & 0.198 \\
\hline
\end{tabular}

Instantaneous vorticity contours and streamlines of flow past the circular cylinder are shown in Fig. 4 at $t=200$. The temporal variation of lift and drag coefficients are shown in Fig. 5. The non-dimensional time ' $t$ ' is obtained by dividing the dimensional time by a factor. Variations of time averaged pressure distribution over the body surface are shown in Fig. 6. The initial convergence pattern of the scheme is shown in Fig. 7. 


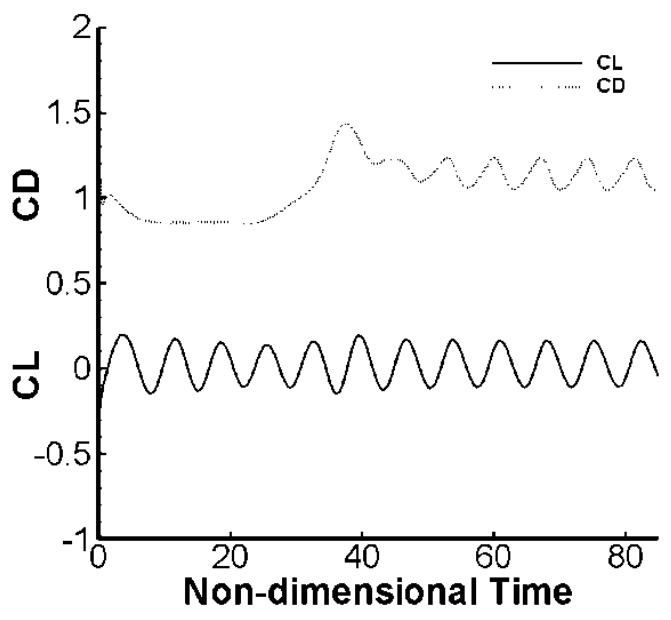

$\operatorname{Re}=100$

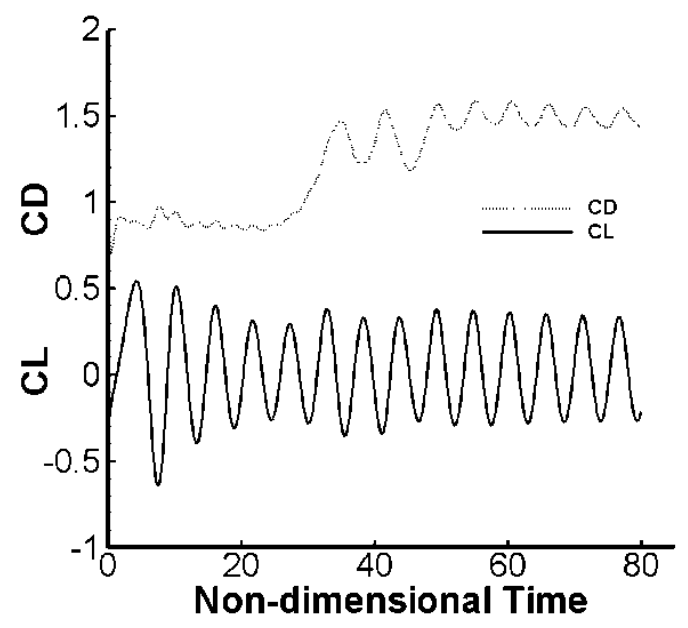

$\mathrm{Re}=200$

Fig. 5. Lift and drag co-efficient for flow past a circular cylinder.

Table 2 Flow parameters around a circular cylinder at $R e=100$ and 200.

\begin{tabular}{|c|c|c|c|c|c|c|}
\hline Parameters & \multicolumn{2}{|c|}{ Drag Coefficient (CD) } & \multicolumn{2}{l|}{ Lift Coefficient (CL) } & \multicolumn{2}{|c|}{ Strouhal number } \\
\hline Reynolds number & $R e=100$ & $R e=200$ & $R e=100$ & $R e=200$ & $R e=100$ & $R e=200$ \\
\hline Belov et al. (1995) & & $1.19 \pm 0.042$ & & \pm 0.64 & $\overline{0}$ & 0.193 \\
\hline Braza et al. $(1986)$ & $1.364 \pm 0.015$ & $1.40 \pm 0.05$ & \pm 0.25 & \pm 0.75 & 0.160 & 0.200 \\
\hline Liu et al. (1998) & $1.350 \pm 0.012$ & $1.31 \pm 0.049$ & \pm 0.339 & \pm 0.69 & 0.164 & 0.192 \\
\hline Ding et al.(2007) & $1.356 \pm 0.010$ & $1.348 \pm 0.05$ & \pm 0.287 & \pm 0.659 & 0.166 & 0.196 \\
\hline Present results & $1.141 \pm 0.094$ & $1.484 \pm 0.05$ & \pm 0.201 & \pm 0.487 & 0.168 & 0.198 \\
\hline
\end{tabular}

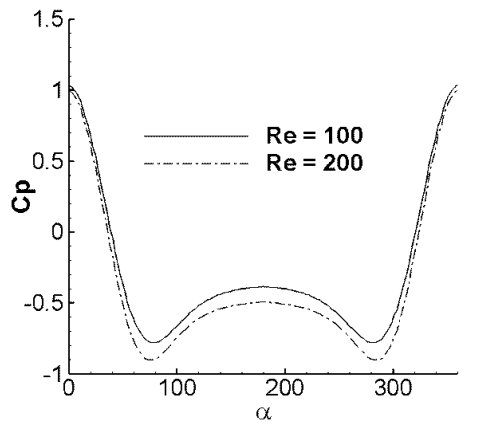

Fig. 6. Variation of time averaged pressure distribution

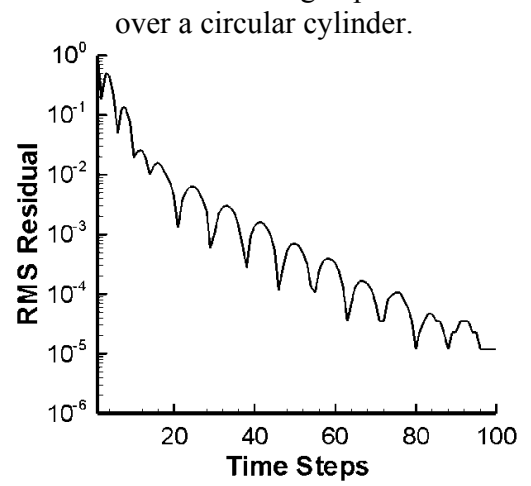

Fig. 7. Initial convergence pattern of the CFR scheme.

Lift and drag coefficients as well as Strouhal number values of the present scheme are compared quantitatively with the results of other researchers. This quantitative comparison is reported in Table 2. It has been observed that our results match well with the results of other researchers found in literature.

\subsection{Numerical Simulation of Flow Past A Circular Cylinder Using Unstructured Grid Based Solver}

In the present study, the unsteady flow at $R e=100$ and 200 are simulated on a triangular mesh with 29,464 cells and 14,878 nodes. The unstructured triangular grid has been generated using GAMBIT 6.2.1 ${ }^{\circledR}$ software and used as input to the unstructured grid based solver. There are 160 nodes on the body as chosen suitably from the grid independence test. The cylinder is placed in a rectangular domain in which the upper and lower boundaries, inlet boundary and outlet boundary are kept at distances of $15 \mathrm{D}, 10 \mathrm{D}$ and $30 \mathrm{D}$ respectively from the centre of the cylinder. The distribution of the cells in the domain is shown in Fig. 3. The non-dimensional time step is set to 0.001 . At every time level, the convergence criteria for pressure-Poisson equation is set in a manner that the residual is less than $10^{-6}$. To obtain the characteristics of lift and drag coefficients, simulation is done upto 400 non-dimensional time.

Figure 8 shows the vorticity contours and streamlines for $R e=100$ and 200 at instantaneous time $t=400$. Figure 9 shows the time dependent behaviour of the lift and drag coefficients on the surface of the cylinder at $R e=100$ and 200 . 


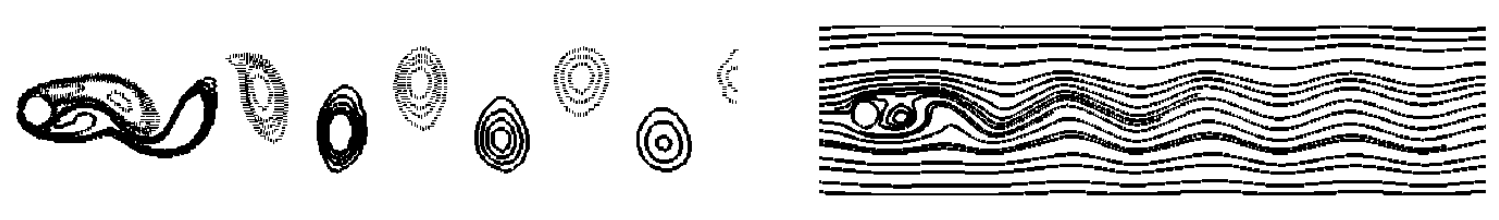

$\mathrm{Re}=100$

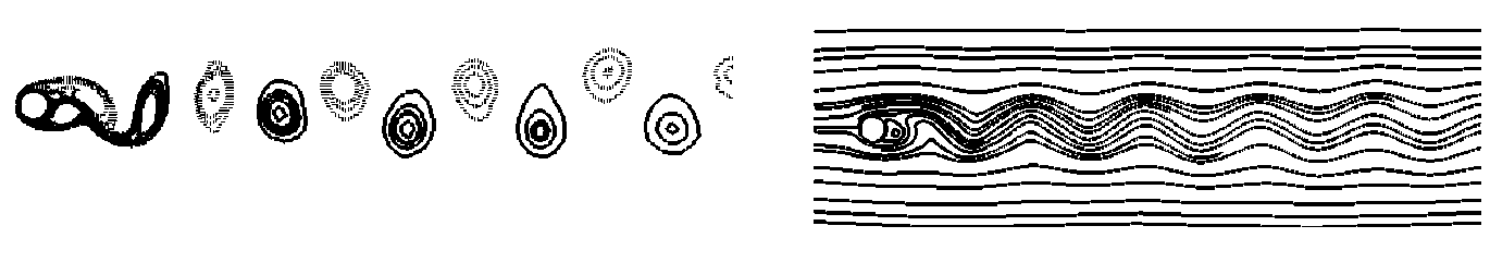

$\mathrm{Re}=200$

Fig. 8. Vorticity contours and streamlines for flow past a circular cylinder.
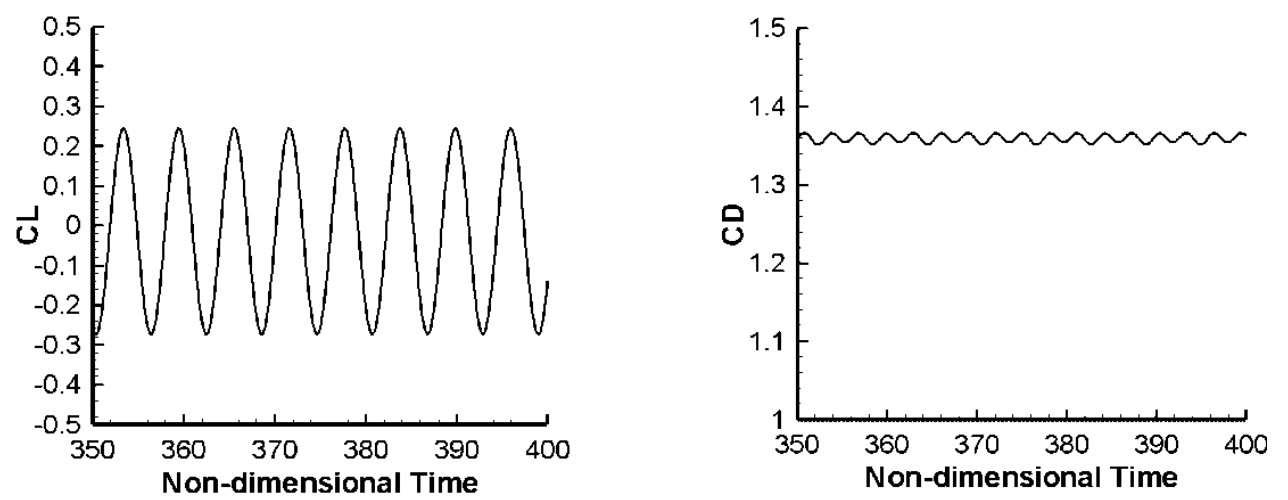

$\mathrm{Re}=100$
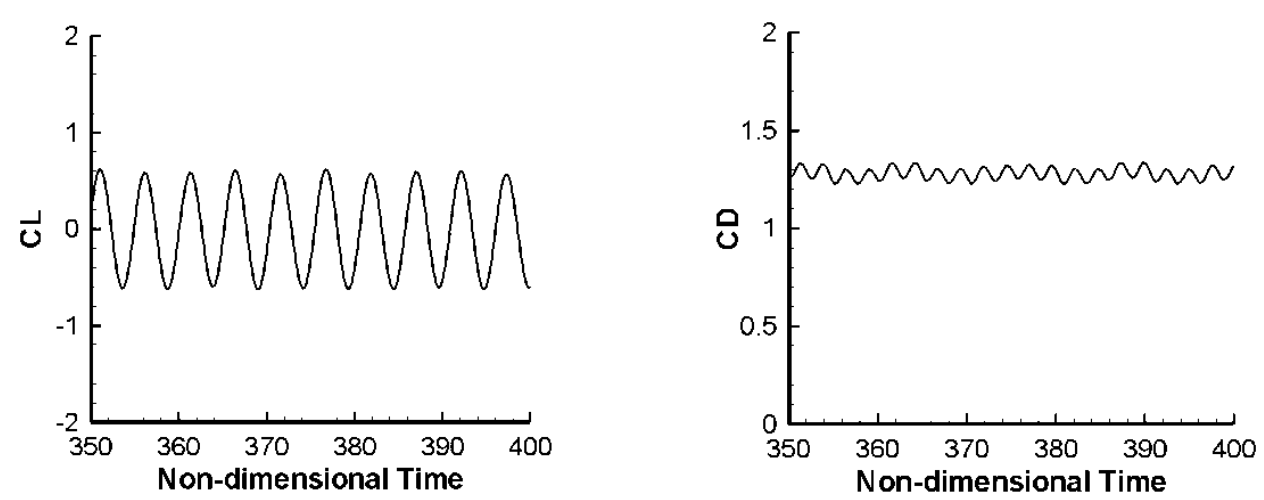

$\operatorname{Re}=200$

Fig. 9. Lift and drag coefficients for flow past a circular cylinder.

Table 3 Flow parameters around one circular cylinder at $R e=100$ and 200.

\begin{tabular}{|l|c|c|c|c|c|c|}
\hline \multicolumn{1}{|c|}{ Parameters } & \multicolumn{2}{|c|}{ Drag coefficient (CD) } & \multicolumn{2}{c|}{ Lift coefficient (CL) } & \multicolumn{2}{c|}{ Strouhal number (St) } \\
\hline \multicolumn{1}{|c|}{ Reynolds number } & $R e=100$ & $R e=200$ & $R e=100$ & $R e=200$ & $R e=100$ & $R e=200$ \\
\hline Meneghini et al. (2001) & $1.370 \pm 0.010$ & $1.30 \pm 0.05$ & -- & -- & 0.165 & 0.196 \\
\hline Ding et al. (2007) & $1.356 \pm 0.010$ & $1.348 \pm 0.05$ & \pm 0.287 & \pm 0.659 & 0.166 & 0.196 \\
\hline Braza et al. (1986) & $1.364 \pm 0.015$ & $1.40 \pm 0.05$ & \pm 0.25 & \pm 0.75 & 0.160 & 0.200 \\
\hline Tritton (1959) & $1.320 \pm 0.010$ & -- & -- & -- & 0.160 & -- \\
\hline Wiesenberger (1921) & $1.326 \pm 0.010$ & -- & -- & -- & 0.1608 & -- \\
\hline Gresho et al. (1980) & $1.816 \pm 0.010$ & -- & -- & -- & 0.18 & -- \\
\hline Present result & $1.352 \pm 0.010$ & $1.32 \pm 0.05$ & \pm 0.278 & \pm 0.602 & 0.161 & 0.192 \\
\hline
\end{tabular}




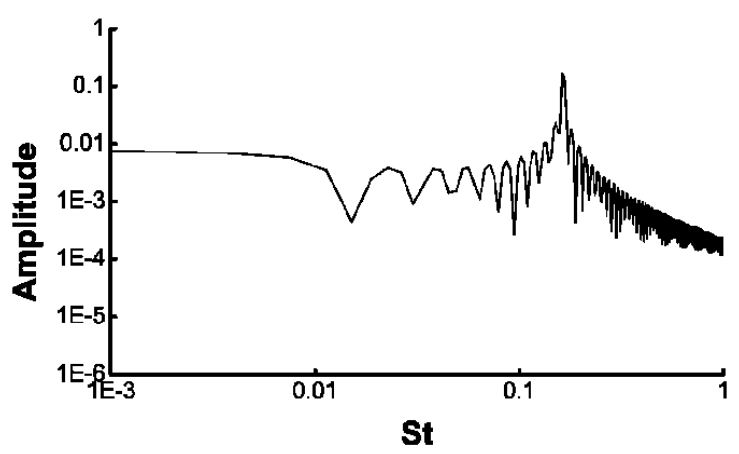

$\mathrm{Re}=100$

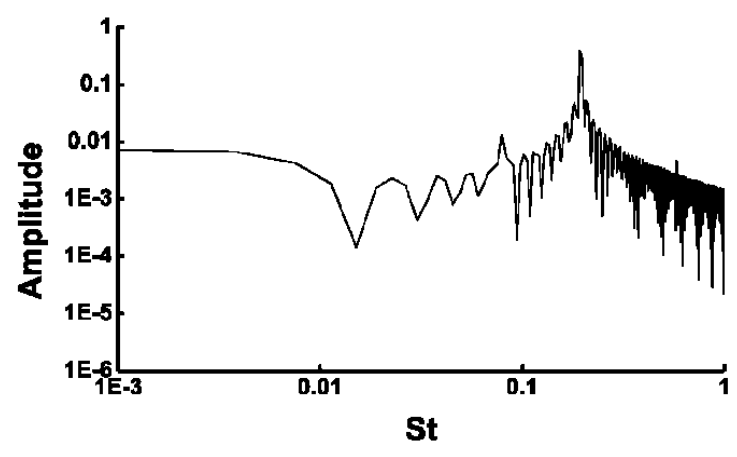

$\mathrm{Re}=200$

Fig. 10. Frequency spectrum of the lift coefficient at $R e=100$ and 200.

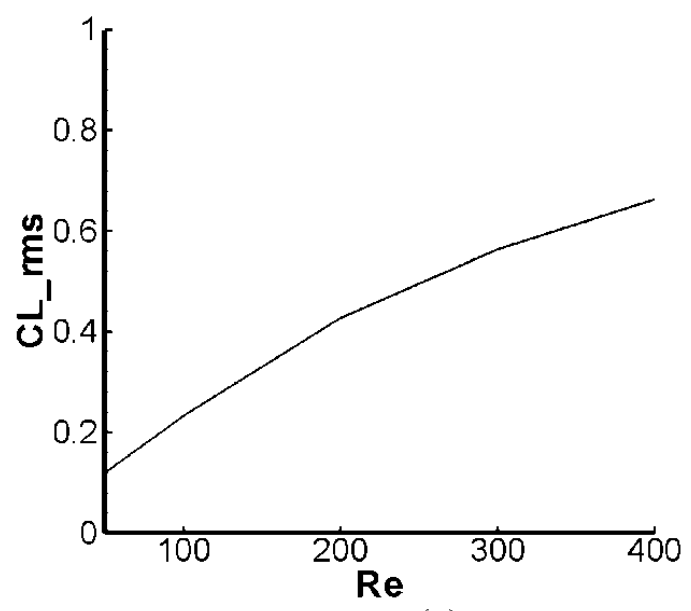

(a)

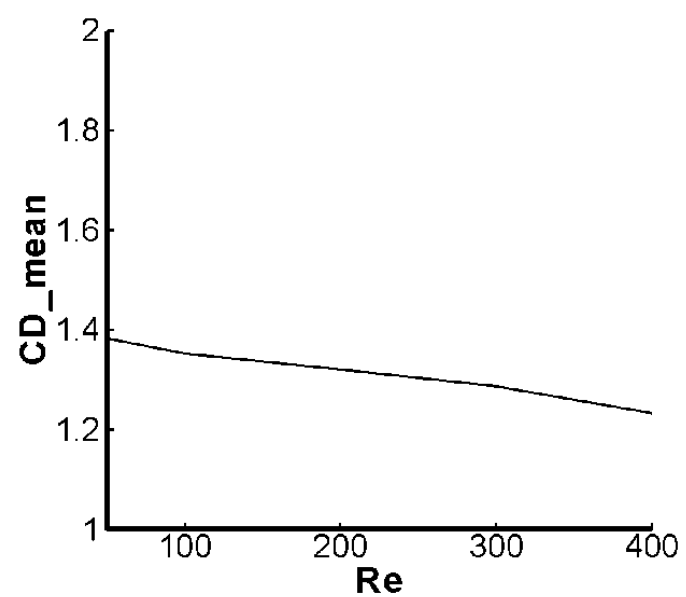

(b)

Fig. 11. Variation of force coefficients with Reynolds number: (a) rms value of fluctuating lift coefficient (b) mean value of fluctuating drag coefficient.

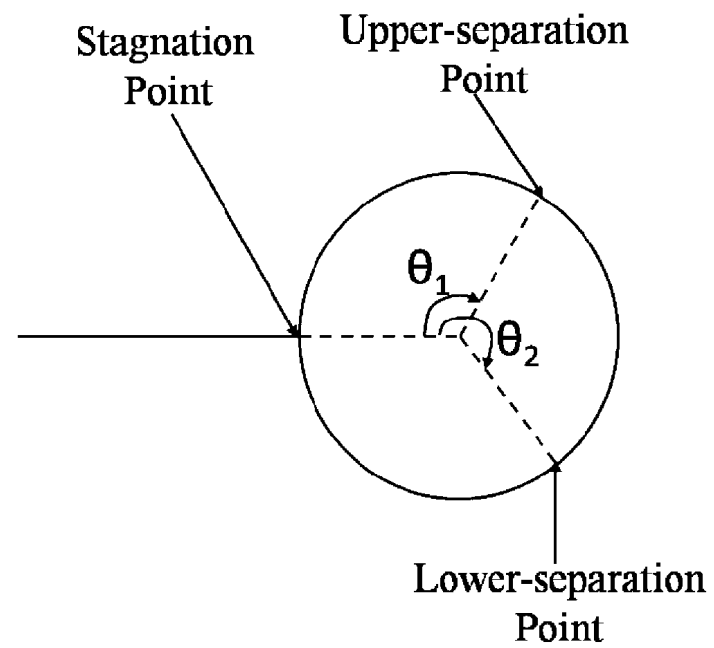

Fig. 12. Definition of stagnation point and separation points.

The clear periodicity illustrated in lift and drag coefficients implies the periodic vortex shedding from the rear surface of the cylinder.

The frequency spectrum of the lift coefficient at $R e=$ 100 and 200 are shown in Fig. 10. The distinct dominating frequency in the frequency spectrum represents the vortex shedding frequency. The rms value of lift coefficient and the mean value of the drag coefficient at different Reynolds number are shown in Fig. 11.

Table 3 lists the Strouhal number, mean value and amplitude of lift and drag coefficients of present results as well as other published results from numerical investigations. It is observed that our results agree very well with those achieved by other researchers. The good agreement of the flow parameters indicates that the CFR method can be employed for further study of flow around multiple circular cylinders and flow past other complex configurations.

It is also observed that unsteady separation of shear layers and their complex interaction in the wake region have profound impact on the time history of stagnation point location and (to a greater extent on) lower and upper separation point locations on the cylinder surface. The stagnation point angle is identified on the basis of highest value of pressure coefficient on the cylinder surface. The location of the pair of separation points is identified on the basis of vanishing wall shear stress. Locations of these points were also obtained based on zero wall vorticity criterion and were found to be very close to those obtained using zero wall shear stress criterion. 

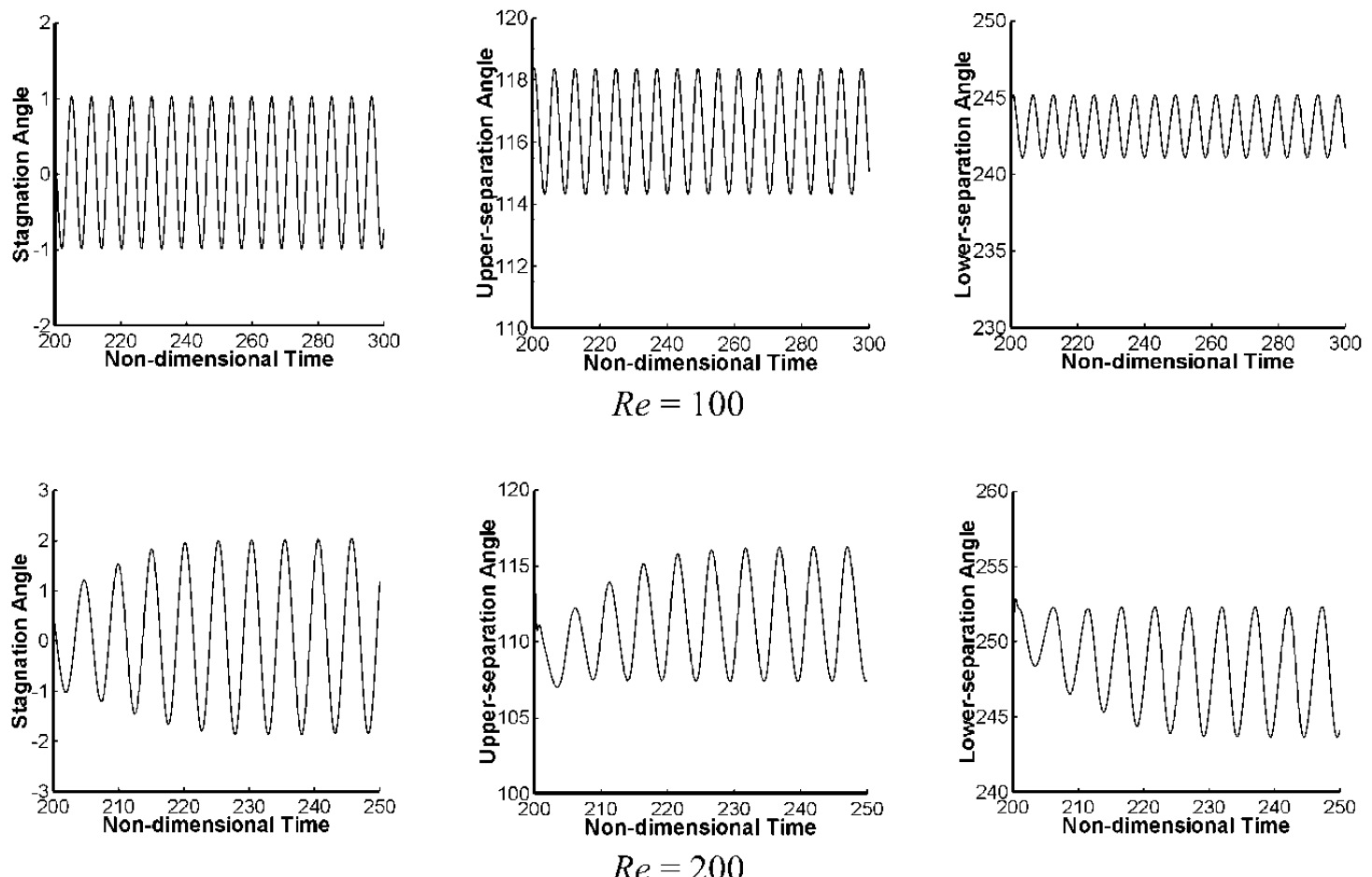

Fig. 13. Time history of stagnation point and separation point angles in degrees.

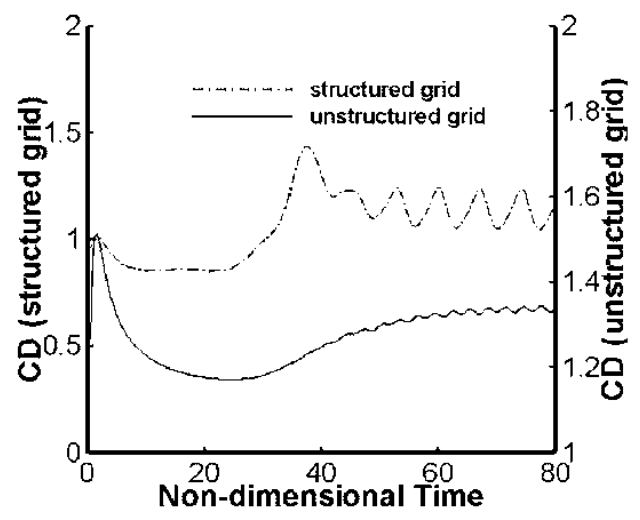

$\operatorname{Re}=100$

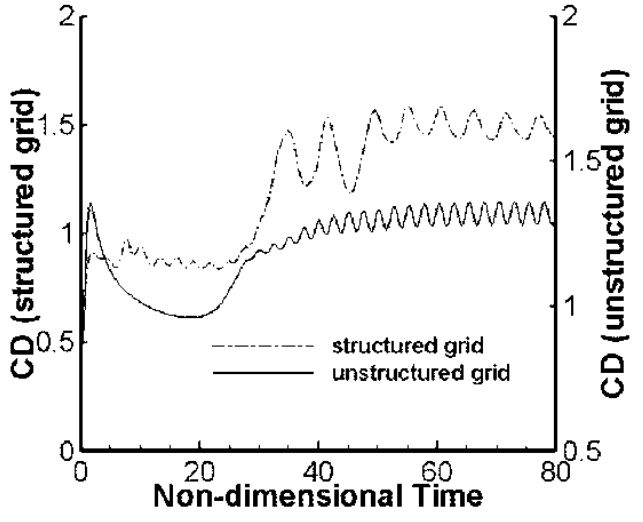

$\operatorname{Re}=200$

Fig. 14. Temporal distribution of drag coefficient with structured and unstructured grid at $R e=100$ and 200 .

The time history of stagnation point angle and upperlower separation point angles for single cylinder is presented in Fig. 13. The angles ' $\theta_{1}$ ' and ' $\theta_{2}$ ' are the angular position of upper and lower separation point measured clockwise from the front stagnation point along the cylinder surface in degrees as shown in Fig. 12. The angles oscillate periodically and the frequency of oscillation is found to be identical to that of the Strouhal frequency which is obtained from FFT of the time history of the lift coefficient for a given configuration.

Figure 14 presents the comparison of temporal behavior of drag coefficients with structured and unstructured grid at $R e=100$ and 200. It is clear that flow transients disappear after a certain number of non-dimensional time steps which is same for both structured and unstructured grid cases. However at $R e=200$, statistically steady flow occurs early as compared to that of flow at $R e=100$.

\subsection{Comparison of the Present Results With Some Experimental Results}

The present numerical results are compared with some of the experimental results from the literature. Figure 15a shows the comparison of the drag coefficients with available experimental and numerical results. It has been observed that our numerical values are generally higher than the experimental values of Tritton (1959) and Weisenberger (1921). Figure 15b shows the comparison of the Strouhal number, which is the dimensionless frequency of vortex shedding, with some of the experimental results of Friehe (1980) and Roshko (1954). 


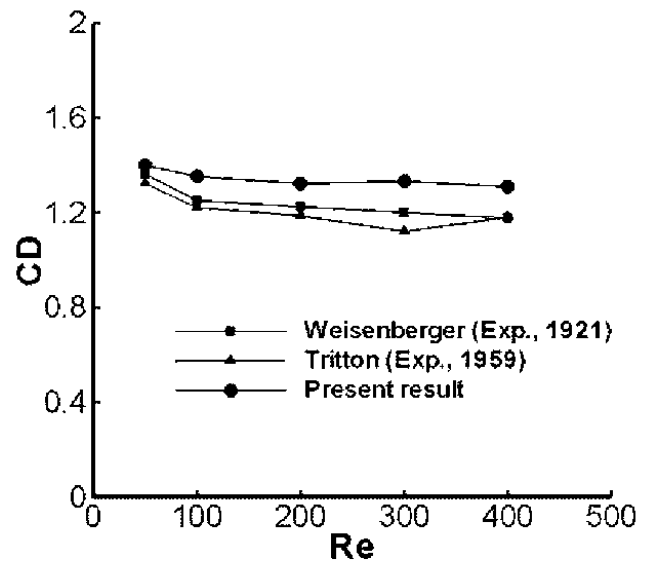

(a)

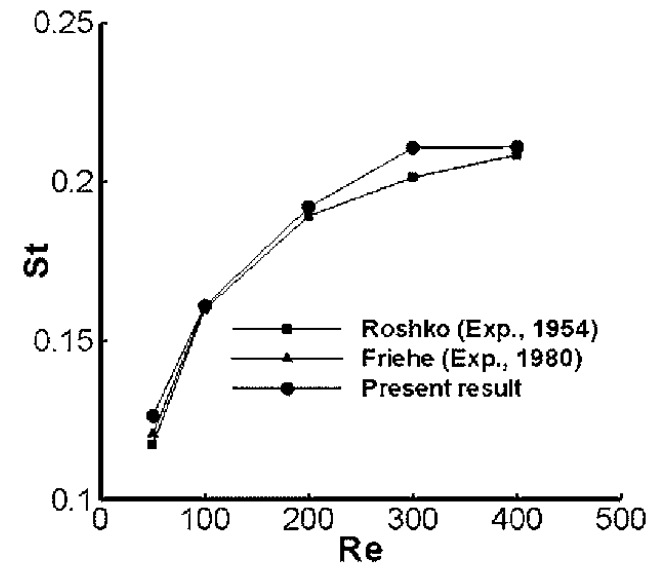

(b)

Fig. 15. Comparison of the present results with other experimental and numerical results.

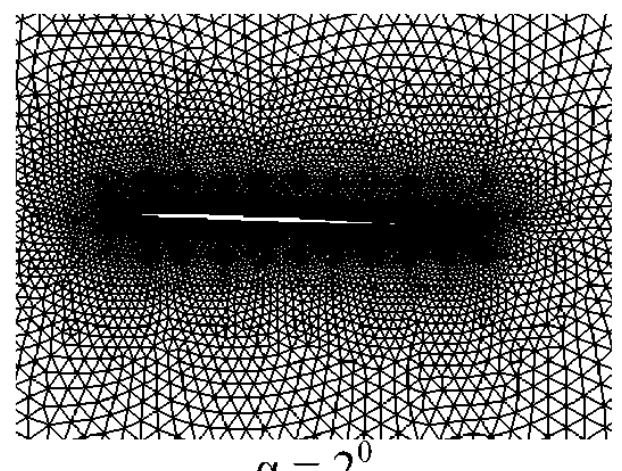

$\alpha=2^{0}$

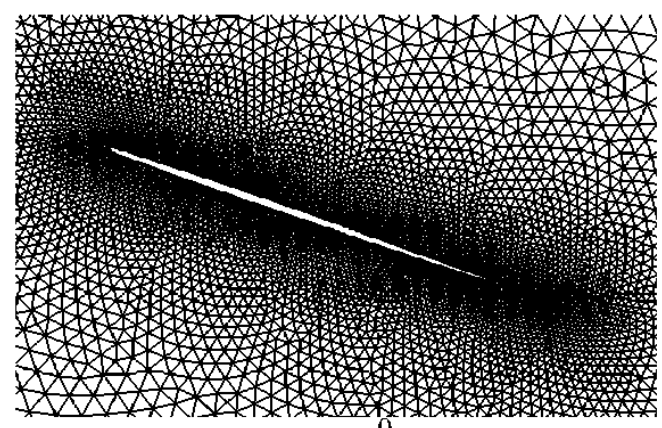

$a=8^{0}$
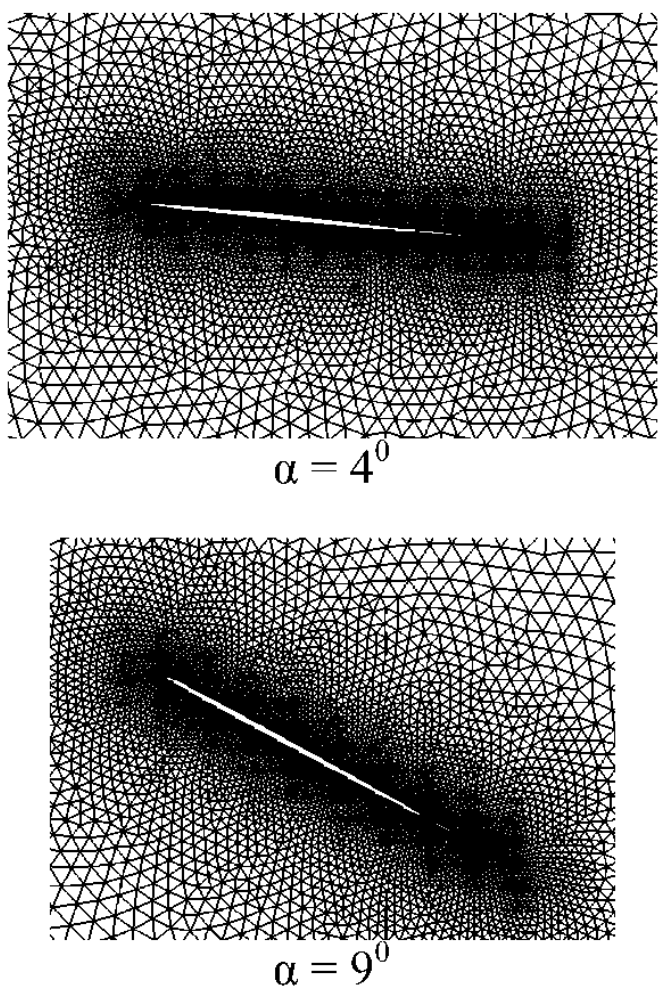

Fig. 16. Triangular mesh around NACA 0002 airfoil at various AOA.

The agreement is seen to be satisfactory. It has been observed that our numerical values are generally higher than the experimental ones. This variation in numerical values for drag coefficients and the Strouhal number from the experimental values is probably due to the relatively coarser grid used downstream of the cylinder.

\subsection{Numerical Simulation of Flow past A NACA0002 Airfoil Using Unstructured}

In the present investigation, an unconfined flow past an NACA 0002 airfoil at various angles of attack (AOA) is calculated at very low Reynolds number on the order of 1000 using CFR solver with unstructured triangular grid. The analysis makes use of two assumptions about the flow field: The flow is incompressible and fully laminar. Incompressibility is well justified for this application as the Mach number will be considerably below 0.3 for a broad range of applications at such ultra-low Reynolds number ranges. The fully laminar flow assumption is more uncertain. In the absence of separation, the flow at these Reynolds numbers will be certainly laminar. Even slight to moderate separation will result in laminar reattachment for chord Reynolds numbers below 10,000 on a smooth airfoil. The degree of separation that might result in transition and the transition length are the unclear issues, but the alternatives are less satisfactory.

The flow field could be assumed fully turbulent, which is surely not the case, or transition could be artificially and rigidly imposed at a specified location. 


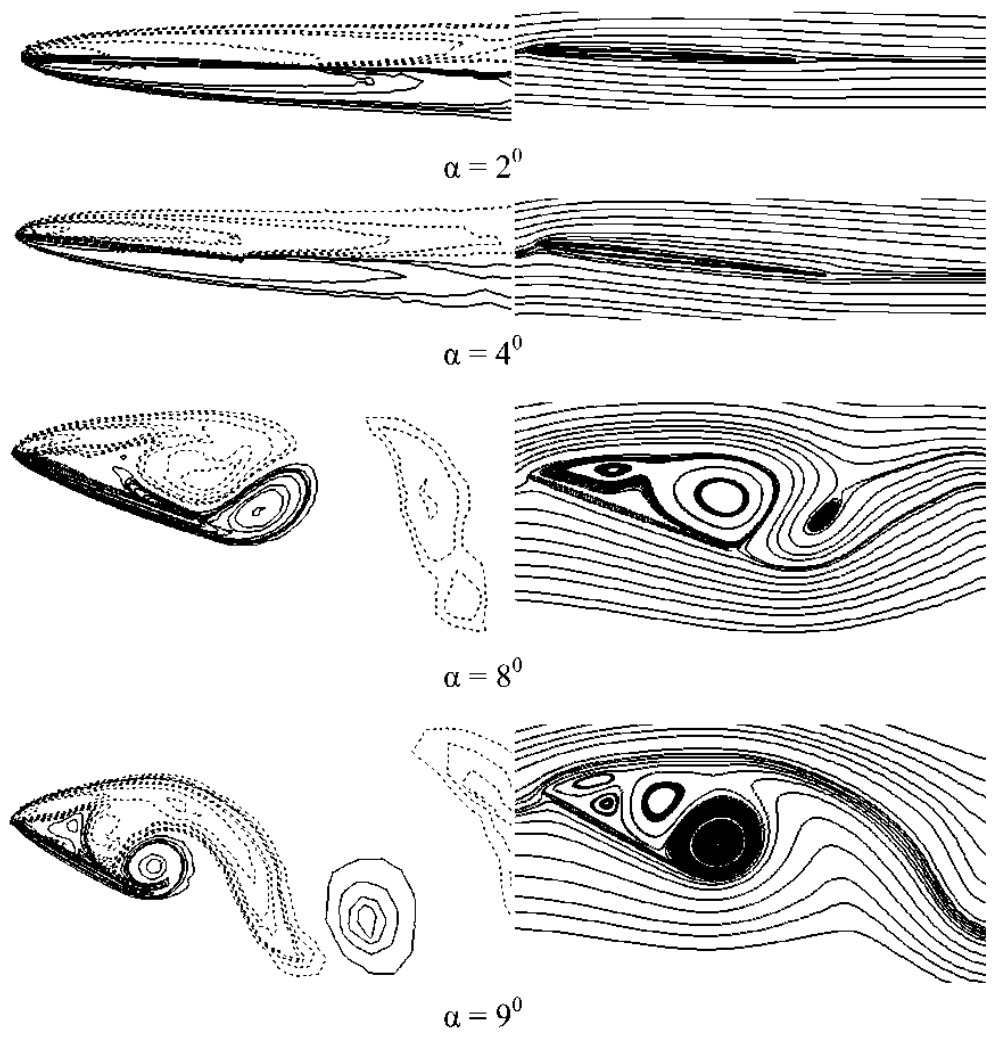

Fig. 17. Vorticity contours and streamlines of flow past NACA 0002 airfoil at various AOA.

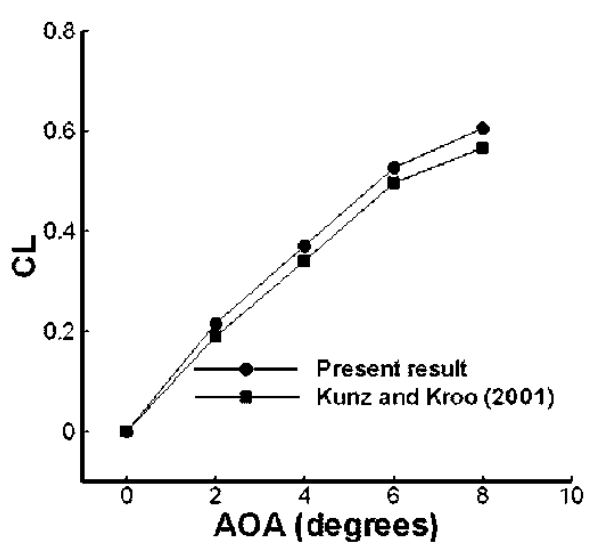

(a)

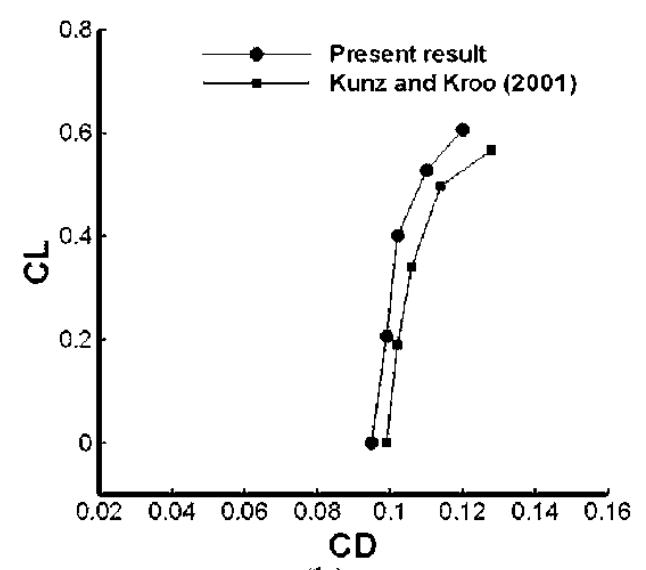

(b)

Fig. 18. (a) Lift curve and (b) Drag polar for the NACA 0002 at $R e=1000$.

Of these three, the fully laminar assumption is less restrictive and most physically accurate (Kunz and Kroo 2001).

In the present study, the triangular meshes generated around NACA 0002 airfoil at various AOA are shown in Fig. 16. The non-dimensional time step used in the calculation is 0.0005. At every time level, the convergence criteria for pressure-Poisson equation is set in a manner that the residual is less than $10^{-6}$.

Figure 17 shows the vorticity contours and streamlines past a NACA 0002 airfoil at various AOA for $R e=$ 1000 at instantaneous time $t=30$. Figure $18 \mathrm{a}$ and $18 \mathrm{~b}$ shows the lift curve and the drag polar curve respectively. The results are reasonably in good agreement with the results reported by Kunz and Kroo (2001). The present result for time averaged surface pressure distribution at $\alpha=0^{0}$ is well compared with the results of Kunz and Kroo (2001) and is shown in Fig. 19. Compared to performance at higher Reynolds numbers, airfoils exhibit an order of magnitude increase in the drag and a similar sized reduction in lift-to-drag ratios. Although the drag rapidly increases as the Reynolds number is reduced, significant lift coefficients are still attainable. Below approximately $R e=1000$, reducing the Reynolds number results in an increase in the maximum steady-state lift. As the Reynolds number is lowered, there is an alleviation of the leading edge suction peak, which results in less adverse gradients along the suction side of the airfoil. 


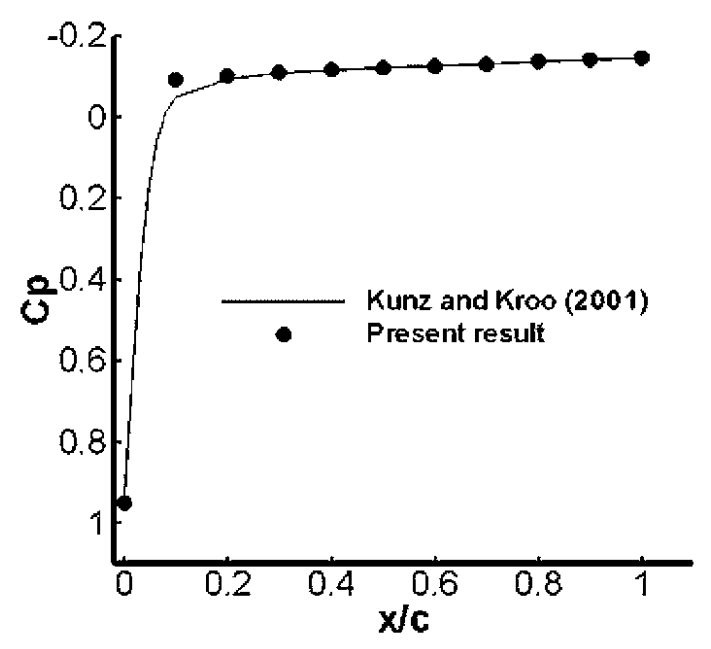

Fig. 19. Time averaged surface pressure distribution on NACA 0002 airfoil at $R e=1000$ and $\alpha=0^{0}$.

This delays separation and allows operation at higher angle of attack.

\section{Conclusion}

The complex problem of unsteady viscous flow is studied at $R e=100$ and 200 around a circular cylinder and at $R e=1000$ around NACA 0002 airfoil over a considerably long non-dimensional time interval. A cell-centered finite-volume scheme has been proposed to solve 2-D Navier-Stokes equations for incompressible viscous flows. Two independent solvers are developed based on a structured O-grid with quadrilateral cells and an unstructured grid with triangular cells. The scheme has been used to study the effect of vortices in laminar flow past a single circular cylinder at two different Reynolds numbers, namely 100 and 200. The two solvers generated results which are comparable to each other. The results show good agreement with computational and experimental results of other researchers. The present scheme demonstrates good convergence characteristics and reasonably good accuracy. The numerical simulations have reestablished the fact that flow past a circular cylinder at moderate Reynolds numbers like 100 and 200 shows interesting features like Karman vortex street formation, time variation of lift and drag forces on the body etc.

For flow past NACA 0002 airfoil at $R e=1000$, flow separation occurs at very small angles of attack $\left(4^{0}\right.$ $\left.6^{0}\right)$. At smaller angles of attack $\left(\alpha \leq 4^{0}\right)$, attached flow is experienced. Since the boundary layer is laminar at $R e=1000$, it is susceptible to separation under mild adverse pressure gradient. At moderate and large angles of attack, large scale separation occurs over a significant portion of the suction side of the airfoil which grossly reduces the effective camber of the airfoil. This reduces the slope of lift curve. No sign of stall is observed within the range of AOA that is used in the present flow case. The drag increases significantly with onset of flow separation. This leads to shallowing of the drag polar curve and consequent reduction of the value of $\mathrm{L} / \mathrm{D}$ ratio and its maximum value. These aerodynamic features need to be studied and accounted well before designing MAVs, UAVs and other ultra low Reynolds number devices.
The results reported in the paper show that both the structured and unstructured versions of the CFR solver are able to satisfactorily capture the features of the flow field past bluff bodies like cylinders and streamlined bodies like airfoils.

\section{ACKNOWLEDGEMENTS}

This work is partially supported by the Air Force Office of Scientific Research, AFRL, USAF, through AOARD Grant Nos. FA2386-08-1-4088 and FA2386-09-1-4109 and by Aeronautics R\&D Board, Ministry of Defence, Government of India research grant, Sanction letter No. DARO/08/1031445/M/I, dated 16.04.2008.

\section{REFERENCES}

Abdallah, S. (1987). Numerical solution for the pressure-Poisson equation with Neumann boundary condition using a non staggered grid. I. Journal of Computational Physics 70, 182-192.

Abdallah, S. (1987). Numerical solution for the pressure-Poisson equation with Neumann boundary condition using a non staggered grid. II. Journal of Computational Physics 70, 193-202.

Belov, A., L. Martinelli and A. Jameson (1995). A new implicit algorithm with multigrid for unsteady incompressible flow calculation. AIAA Journal 95(9).

Braza, M., P. Chassaing, and M.H. Ha (1986). Numerical study and physical analysis of the pressure and velocity fields in the near wake of a circular cylinder. Journal of Fluid Mechanics 163, 79-130.

Chakrabartty, S.K. (1990). Vertex-based finite-volume solution of the two-dimensional Navier-Stokes Equations. AIAA Journal.

Cheng, M., G.R. Liu (2000). Effects of after body shape on flow around prismatic cylinders. Journal of Wind Engineering and Industrial Aerodynamics 84, 181-196.

Choi, S.K., H.Y. Nam and M. Cho (1993). Use of the Momentum Interpolation Method for numerical solution of incompressible flows in complex geometries: choosing cell face velocities. Numerical Heat Transfer Part B 23, 21-41.

Dalal, A., V. Eswaran and G. Biswas (2008). A finitevolume method for Navier-Stokes equations on unstructured meshes. Numerical Heat Transfer Part B 54, 238-259.

Deng, G.B., J. Piquet, P. Queutey and M. Visonneau (1994). Incompressible flow calculations with a Consistent Physical Interpolation -Finite Volume Approach. Computers and Fluids 23(8), 10291047.

Ding, H., C. Shu, K.S. Yeo and D. Xu (2007). Numerical simulation of flows around two circular 
cylinders by mesh-free least square-based finite difference methods. International Journal for Numerical Methods in Fluids 53, 305-332.

Friehe, C.A. (1980). Vortex shedding from cylinders at low Reynolds numbers. Journal of Fluid Mechanics 100, 237-241.

Gresho, P.M., S.T. Chan, R.L. Lee and C.D. Upson (1980). On the time dependent solution of the incompressible Navier-Stokes equations. Recent Advances in Numerical Methods in Fluids, Pineridge Press, Swansea.

Kang, S. and Y. Kim (2002). Pressure-based unstructured grid finite volume method for simulating laminar reacting flows. Numerical Heat Transfer Part. B 41, 53-72.

Liu, C., S. Zheng and C.H. Sung (1998). Preconditioned multigrid methods for unsteady incompressible flows. Journal of Computational Physics 139, 21-35.

Mathur, S.R., and J.Y. Murthy (1997). A pressurebased method for unstructured meshes. Numerical Heat Transfer Part B 31, 195-215.

Mathur, S.R., and J.Y. Murthy (1997). Pressure boundary conditions for incompressible flow using unstructured meshes. Numerical Heat Transfer Part B 32, 283-298.

McBride, D., N. Croft and M. Cross (2007). Finite volume method for the solution of flow on distorted meshes. International Journal of Numerical Methods for Heat and Fluid Flow 17(2), 213-239.

Meneghini, J.R., F. Saltara, C.L.R. Siqueira and J.A. Ferrari Jr. (2001). Numerical simulation of flow interference between two circular cylinders in tandem and side-by-side arrangement. J. of Fluids and Structures 15, 327-350.

Nakamura, Y., Y. Ohya, S. Ozono and R. Nakayama (1996). Experimental and numerical analysis of vortex shedding from elongated rectangular cylinder at low Reynolds number 200-103. Journal of Wind Engineering and Industrial Aerodynamics 65, 301-308.

Nishikawa, H. (2007). Multigrid third-order leastsquares solution of Cauchy-Riemann equations on unstructured triangular grids. International Journal for Numerical Methods in Fluids 53, 443454.

Perron, S., S. Boivin and J.M. Herard (2004). A finite volume method to solve the $3 \mathrm{D}$ Navier-Stokes equations on unstructured collocated meshes. Computers and Fluids 33, 1305-1333.

Perry, A.E., M.S. Chong and T.T. Lim (1982). The vortex-shedding process behind two-dimensional bluff bodies. Journal of Fluid Mechanics 116, 7790 .

Raithby, G.D. (1999). Discussion of the finite-volume method for radiation and its application using 3D unstructured meshes. Numerical Heat Transfer Part. B 35, 389-405.

Rhie, C.M. and W.L. Chow (1983). Numerical study of turbulent flow past an airfoil with trailing edge separation. AIAA Journal 21(11).

Roshko, A. (1954). On the drag and shedding frequency of two dimensional bluff bodies. NACA TN, 3169 .

Roy, A. and G. Bandyopadhyay (2006). A finite volume method for viscous incompressible flows using a consistent flux reconstruction scheme. International Journal for Numerical Methods in Fluids 52, 297-319.

Sang, W. and F. Li (2007). An unstructured/structured multi-layer hybrid grid method and its application. International Journal for Numerical Methods in Fluids 53, 1107-1125.

Tritton, D.J. (1959). Experiments on the flow past a circular cylinder at low Reynolds numbers. Journal of Fluid Mechanics 6, 547.

Wiesenberger, V.C. (1921). Neuere festellungen über die Gesetze des Flüssigkeits und Luftwiderstands. Physik. Z. 22, 231.

Woodfield, P.L. and K. Suzuki (2003). Performance of a three-dimensional, pressure-based, unstructured finite-volume method for low Reynolds number incompressible flow and wall heat transfer rate prediction. Numerical Heat Transfer Part B 43, 403-423. 